\title{
Quantifying Interference in WiLD Networks using Topography Data and Realistic Antenna Patterns
}

\author{
Michael Rademacher \\ Hochschule Bonn Rhein-Sieg \\ Sankt Augustin, Germany \\ michael.rademacher@h-brs.de
}

\author{
Mathias Kretschmer \\ Fraunhofer FIT \\ Sankt Augustin, Germany \\ mathias.kretschmer@fit.fraunhofer.de
}

\author{
Karl Jonas \\ Hochschule Bonn Rhein-Sieg \\ Sankt Augustin, Germany \\ karl.jonas@h-brs.de
}

\begin{abstract}
Avoiding possible interference is a key aspect to maximize the performance in Wi-Fi based Long Distance networks. In this paper we quantify self-induced interference based on data derived from our testbed and match the findings against simulations. By enhancing current simulation models with two key elements we significantly reduce the deviation between testbed and simulation: the usage of detailed antenna patterns compared to the cone model and propagation modeling enhanced by license-free topography data. Based on the gathered data we discuss several possible optimization approaches such as physical separation of local radios, tuning the sensitivity of the transmitter and using centralized compared to distributed channel assignment algorithms. While our testbed is based on $5 \mathrm{GHz}$ Wi-Fi, we briefly discuss the possible impact of our results to other frequency bands.
\end{abstract}

Index Terms-Interference, Directional antennas, 802.11, WiFi, Simulation, Measurement

\section{INTRODUCTION}

To date, only one out of two people world-wide and only one out of seven people in the Least Developed Countries (LDC) are using the Internet [1]. As the cost to provide broadband connectivity in sparsely populated low-income regions are seen as a major obstacle so-called "Alternative Networks" are being evaluated [2]. The majority of these technologies are wireless solutions which overcome the costly process of deploying fiber. One of these alternatives, WiFibased Long Distance (WiLD) networks use Commercial Offthe-Shelf (COTS) high-gain directional antennas and IEEE 802.11 (Wi-Fi) transmitters to span wireless Point To Point (P2P) links in a multi-hop topology at distances up to $25 \mathrm{~km}$ per hop. Due to the distribution in the consumer sector, Wi-Fi transmitters feature a low energy design and provide a stable performance in unlicensed frequency bands. WiLD networks are mainly used in the backhaul segment providing the intermediate links between the backbone and the access networks at the edge. To maximize the capacity and exhibit characteristics of a carrier-grade network intelligent Channel Assignment (CA) and traffic-engineering techniques [3] are applied.

Similar to other wireless technologies, avoiding possible interference is one element to maximize the performance in WiLD networks. Despite using high-gain directional antennas, we experienced a significant amount of self-induced (internal) interference in our testbed which motivated this work. To gain additional insights and quickly adapt different parameters we decided to conduct a simulation based study. We replicated our testbed in a simulation. However, first results showed a significant difference between simulation and reality. Therefore, we decided to further analyze the required steps to optimize the simulation.

In Section II we summarize background information and related work. In Section III we provide an extended methodology including the description of our testbed and the simulation model. Section IV describes our results and Section V is a conclusion while Section VI emphasizes future work items.

\section{BACKGROUND AND RELATED WORK}

a)
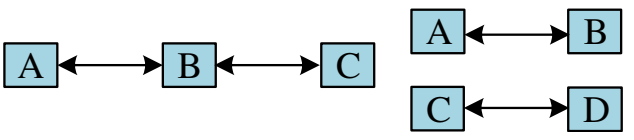

c)

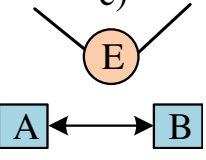

Fig. 1: Different types of interference: (a) Intra-flow (b) Interflow (c) External interference [4].

We use the interference taxonomy illustrated in Fig. 1. Intra-flow interference (Fig. 1a) occurs when one node (B) communicates with two neighboring nodes $(\mathrm{A}, \mathrm{C})$ on the same channel. Inter-flow interference occurs between two links from different paths but in the same interference range (Fig. 1b). External interference occurs due to other participants transmitting on the same channel. We have studied external interference in [5] and focus on intra-flow and inter-flow interference in this work.

In our network architecture each node is equipped with multiple Wi-Fi transmitters. Each of them is connected to an individual directional antenna. To form the desired topology the antennas were manually aligned to a neighboring node during the build-up phase of the network. The topology is static with no mobility and changing desired links (alignment of the antennas) involves manual labor. We focus on the $5 \mathrm{GHz}$ U-NII band which provides significantly more bandwidth $(500 \mathrm{MHz})$ and allows a higher transmission power (regulation) compared to the $2.4 \mathrm{GHz}$ ISM band.

Mobile Adhoc Networks (MANETs) equipped with omnidirectional antennas have been extensively studied in simulations 
and testbeds. Joint studies for $\mathrm{Wi}-\mathrm{Fi}$ radios with directional antennas are rare. A comprehensive overview of different simulation based studies using directional wireless communication is provided in [6]. Similar to this work, the authors in [7] describe interference measurement in a metropolitan multiradio testbed with link distances from $1 \mathrm{~km}$ to $5 \mathrm{~km}$. However, the focus of their work is the impact of interference to network performance.
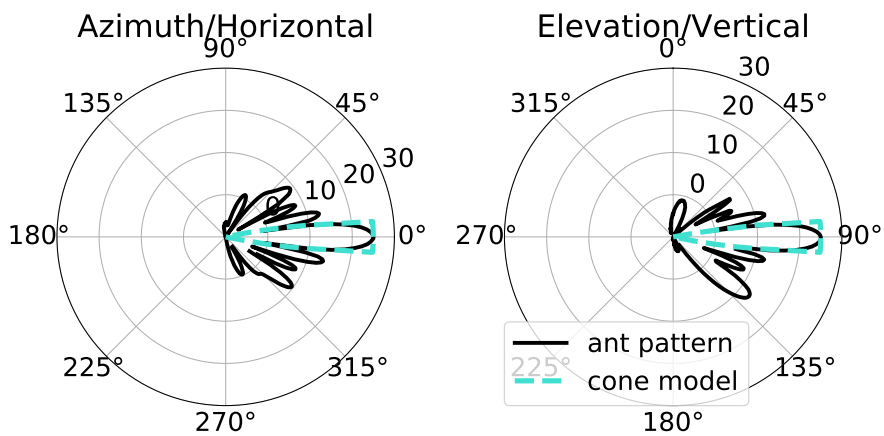

Fig. 2: Pie-slice/cone model of interference [8] compared to the actual antenna pattern used in this work.

The so-called cone (3D) or pie-slice (2D) interference model for directional antennas is, due to its simplicity, widely used in the context of directional Wireless Mesh Networks (WMNs) [8], [9], [10], [11]. In addition, the model is also applied to mmWave and Terahertz Communication Systems [12]. Fig. 2 illustrates an example as an overlay to the antenna pattern we used in this work. The main limitation of this model is the missing representation of side- or back-lobes. The authors in [13] comprehensively highlight that the pie-slice model exaggerates the throughput attained in ad-hoc networks. In [14], we conducted a simulation based study to analyze the effects of side- and back-lobes to the performance of the Wi-Fi MAC-layer.

To the best of our knowledge, this is the first contribution which analyzes internal interference in a WiLD network using a simulation based approach verified by testbed measurements.

\section{Methodology}

The topologies in the simulation and testbed are identical. They consists of $\mathrm{N}=12$ nodes with $\mathrm{R}=21 \mathrm{Wi}-\mathrm{Fi}$ radios (IEEE $802.11 \mathrm{n}$ ). We use two types of antennas with $19 \mathrm{dBi}$ and $25 \mathrm{dBi}$ gain respectively. Latter is illustrated in Fig. 2. The topology is visualized in Fig. 3a and consists of P2P-links with distances ranging between a few hundred meters up to $10.3 \mathrm{~km}$. Packets are routed along the predefined tree-topology originating from a single gateway. The multiple antennas at a single location are mounted on a pole with small distances in between (cf. Fig. 3b). Depending on the location of the neighboring nodes there exists a variable angle between the antennas.

We represent the overall network interference as a matrix $A$ of size $R * R$ where $A(i, j)$ depicts the signal strength in $\mathrm{dBm}$ originating from the $i^{\text {th }}$ radio received by the $j^{\text {th }}$ radio. The value from a radio to itself $(A(i, i))$ is undefined.
In addition, based on the topology, the interference matrix includes desired links which are not considered as interference. If two radios are mounted on the same Single-Board Computer (SBC), the main factor for interference is crosstalk within the enclosure. Based on additional experiments, we account for this by setting all crosstalk radio pairs to a static value of $-50 \mathrm{dBm}$ in the simulation and for the testbed measurements. This value represents a lower estimate, depending on the positions on the SBC and Wi-Fi transmitter this value can be even higher.

Our testbed is located in the Rhein-Sieg area of Germany around the Fraunhofer Campus in Sankt Augustin. Instead of relying solely on radio-towers, we focus on a practical build-up by mainly exploiting rooftops. Our WiLD nodes consist of a SBC with multiple Wi-Fi cards plugged into (mini-)PCI-e slots. We use three types of Wi-Fi cards based on the same chip and using the same Linux driver (ath9k). The SBCs are placed in outdoor suitable enclosures mounted at the desired places (cf. Fig 3b). The testbed is orchestrated by our WiLD network management software called WiBACK [3]. WiBACK manages the connectivity to each node and a CA algorithm aims to avoid interference. However, to obtain the desired interference matrix, we disabled the CA algorithm and used the same channel on each radio. To account for signal fading effects, we collected 8000 single measurements over a time period of 8 hours evenly distributed over all (23) available $20 \mathrm{MHz}$ channels in the U-NII band. The signal strength measured in our testbed has a lower bound at the Clear Channel Assessment (CCA) threshold of $-100 \mathrm{dBm}$. We obtained the desired interference matrix $A_{T}$ by averaging all single measurements from our testbed.

We use the de facto standard simulator for academic research, network simulator 3 (ns-3) in the current version 3.28, due to its modularity and extendability. We only focus on the important aspects in this section and refer the reader to the supplement source-code on our website [15]. After evaluating first results, we decided to enhance the simulation with two modules to increase the similarity to our testbed.

The first enhancement is a class which is able to load and use antenna radiation pattern. We implemented a generic approach called FileAntenna module using the ".ant" v3 data format provided by antenna vendors. Additional information about this enhancement can be found in [14].

For line-of-sight links with sufficient Fresnel zone clearance, estimating the path-loss using the well-known FreeSpace Path Loss (FSPL) equation is a viable approach. This is the case for all communication links in our network. However, our goal is to evaluate interference between all radios and landmarks may block the line-of-sight path for two interfering radios. Therefore, we added topography data to enhance the propagation modeling in $\mathrm{ns}-3$. We computed the propagation loss from each radio to each radio including the elevation above ground before the simulation starts. We selected the Longley-Rice model (also known as the irregular terrain model) [16] based on the results in [17]. This model includes diffraction and ground reflection effects and is mainly used 


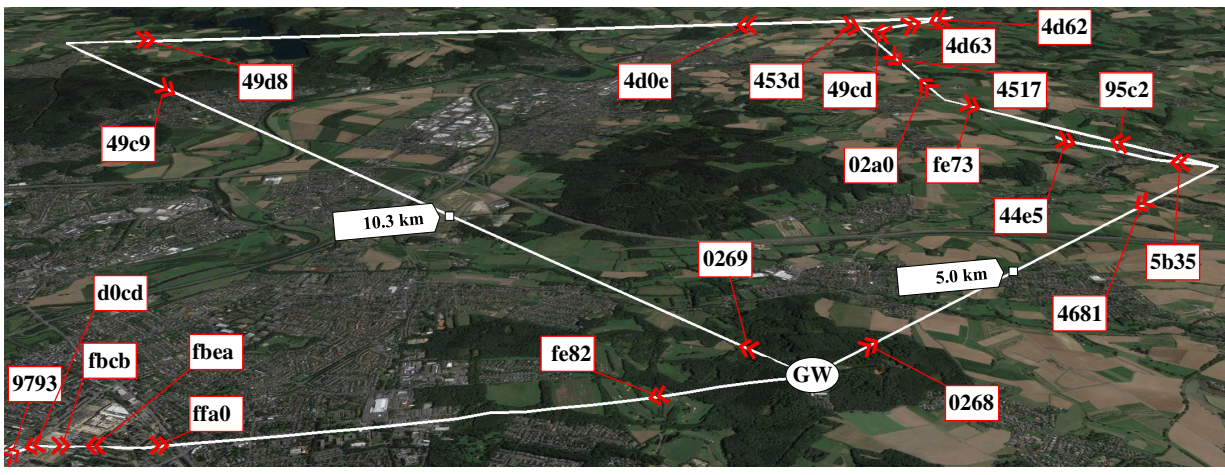

(a) Measured links. Image credit: Landsat / Copernicus. Imagery Date 08/24/2016.

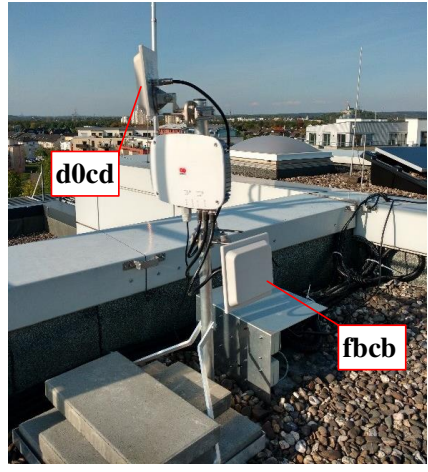

(b) Example of a node.

Fig. 3: Our WiLD Testbed. Location of Gateway: N50 $44^{\prime} 58.46^{\prime \prime}$, E7 $7^{\circ} 12^{\prime} 14.11^{\prime \prime}$.

for television broadcasting $(<1 \mathrm{GHz})$ but also suitable for our frequency bands. The required topography data has been acquired by the Shuttle Radar Topography Mission (SRTM). The data is freely available online [18] at a resolution of 1 arc second $(\approx 30 \mathrm{~m})$ between $60^{\circ} N$ and $57^{\circ} S$ [19]. In addition, we implemented the possibility to import nodes with their GPS coordinates making it more convenient to replicate our testbed in the simulation. We used the last digits of the MAC address as a common identifier for the corresponding radios. The routing, transmission power and modulation in the experiments are static to focus on the interfering signal power. After running the simulation including our extensions for the model for a sufficient duration to stabilize the protocols used, we obtained the desired interference matrix $A_{S}$ in the same format as $A_{T}$.

\section{RESUlTS}

Based on the harvested data represented by the interference matrices $A_{T}$ and $A_{S}$, the first part of this section deals with a comparison between both methodologies. Afterwards, we analyze selected aspects of internal interference more precisely.

\section{A. Simulation vs. Testbed}

Both interference matrices $A_{T}$ and $A_{S}$ are visualized in Fig. 4a and Fig. 4b respectively. The heat map reveals the interference signal power, measured or simulated, from each radio to each radio. The communication links are marked with the letter "L". Both heat maps visualize significant similarities indicating the developed extensions provide an improvement in simulation accuracy. We will further quantify this in the following.

To provide a meaningful comparison between the developed extensions, we decided to transform both interference matrices into a binary format. Each signal level above the CCA threshold leads to a channel busy state (interference) at the Wi-Fi MAC layer to assert fair access to the medium in a distributed way. Therefore, a binary classification for the number of interference above this threshold is one possible representation for this work. We conducted the transformation in the following way:

$$
A^{\prime}(i, j)= \begin{cases}1, & \text { for } A(i, j) \geq-100 \mathrm{dBm} \\ 0 & \text { for } A(i, j)<-100 \mathrm{dBm}\end{cases}
$$

A comparison between $A_{T}^{\prime}$ and $A_{S}^{\prime}$ can be conducted based on a difference matrix $A_{D}^{\prime}=A_{T}^{\prime}-A_{S}^{\prime}$. The values in $A_{D}^{\prime}$ represent the simulation error using three different cases.

- 0 , if the binary interference is present in the testbed and the simulation (white in Fig. 5).

- -1 , if the binary interference is present in the simulation but not in the testbed (black in Fig. 5).

- 1 , if the binary interference is present in the testbed but not in the simulation (grey in Fig. 5).

Since both errors are equally undesirable, the overall goal is to minimize the sum off all elements of $A_{D}^{\prime}$ squared.

$$
\epsilon=\frac{\sum_{i, j=1}^{R} A(i, j)^{2}}{R^{2}}
$$

To point out the impact of our developed extensions, we conducted four different simulations and visualized $A_{D}^{\prime}(i, j)$ individually in Fig. 5.

Fig. 5a is based on a simulation using the FSPL equation and the cone model representing the state of the art prior to this work. The simulation significantly underestimates the interference since the cone model does not consider any side- or back-lobes. There exists a significant deviation of $\epsilon=0.215$.

Fig. $5 \mathrm{~b}$ is based on a simulation using the Longley-Rice model and the cone interference model. The result is identical to the previous simulation with a deviation of $\epsilon=0.215$. There are two reasons for the identical result. First, the cone interference model only considers the main-lobe of the antenna and second, the antennas in our testbed are aligned and the main-lobe points to a direct neighbor at a line-of-sight path. Therefore, using topography data does not decrease the error compared to the FSPL equation.

Fig. $5 c$ is based on a simulation using the FSPL equation and our detailed antenna model. The overall deviation halves to 


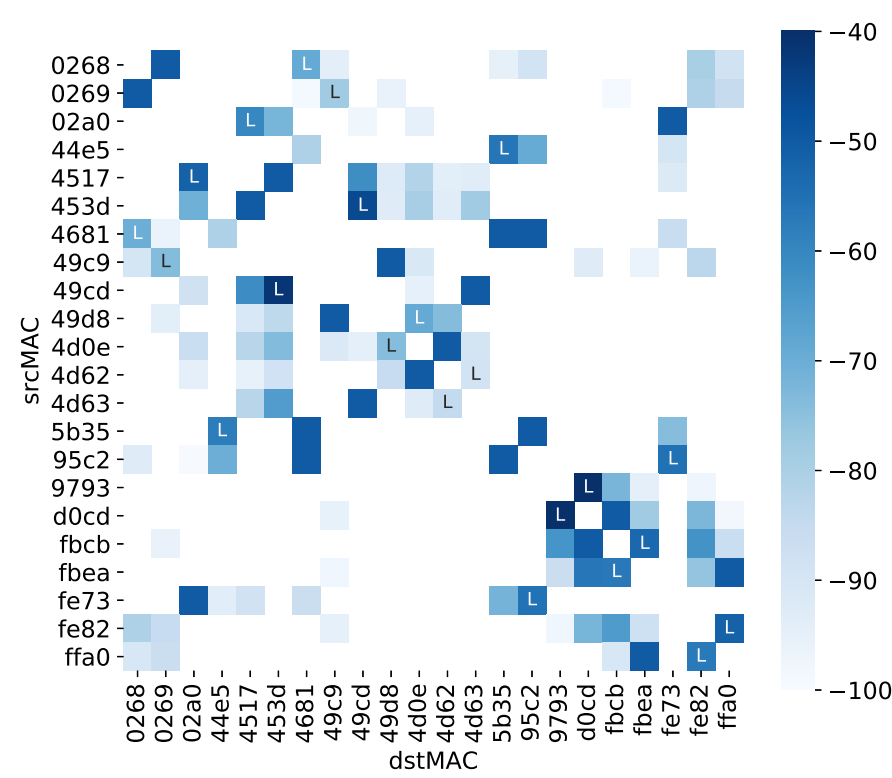

(a) $A_{T}$ based on the testbed.

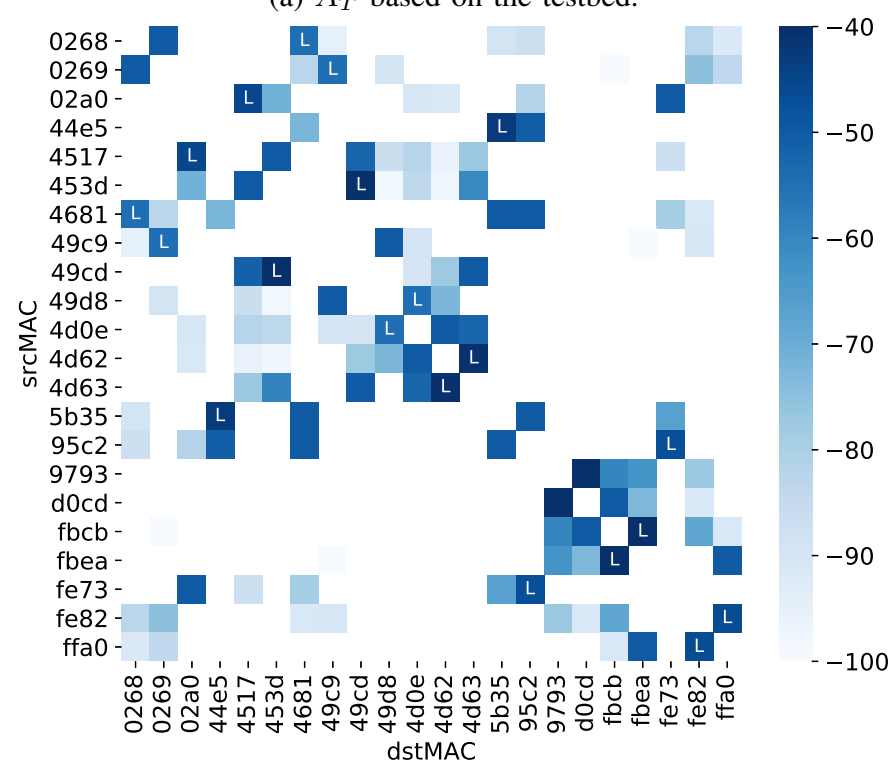

(b) $A_{S}$ based on the final simulation.

Fig. 4: Heat map of the interference matrices.

$\epsilon=0.10$. The vast majority of interfering links in the testbed are now estimated correctly by the simulation. This indicates a significant amount of internal interference originating from the side- and back-lobes of our antennas. However, this simulation still uses the FSPL model, and therefore the simulation now overestimates the internal interference.

Fig. 5d reveals the final results using the Longley-Rice model (topography data) and antenna patterns. This figure is the binary representation of the deviation between Fig. 4a and Fig. 4b. The overall error significantly decreases to $\epsilon=0.033$. In other words, our simulation is capable to predict interference with $97 \%$ accuracy. The remaining error is distributed equally over both possible errors. We conducted a first individual analysis visualized in Fig. 5d to gain further

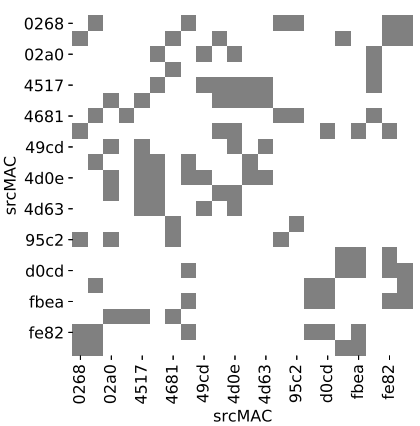

(a) FSPL and cone model: $104(21.5 \%)$ in testbed but not in the simulation.

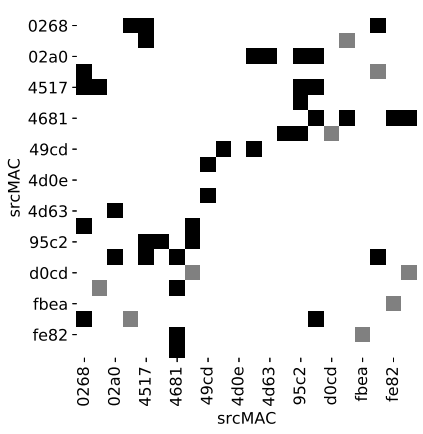

(c) FSPL and antenna pattern: $9(1.9 \%)$ in testbed but not in simulation, $39(8.1 \%)$ in simulation but not in testbed.

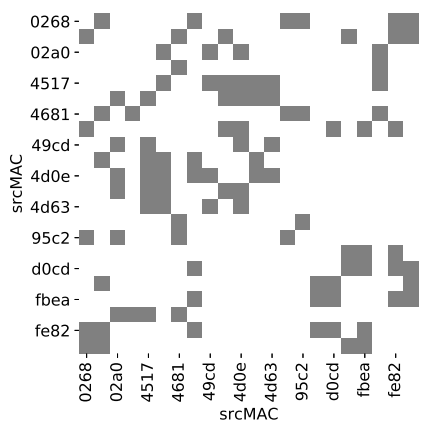

(b) Topography data and cone model: $104(21.5 \%)$ in testbed but not simulation.

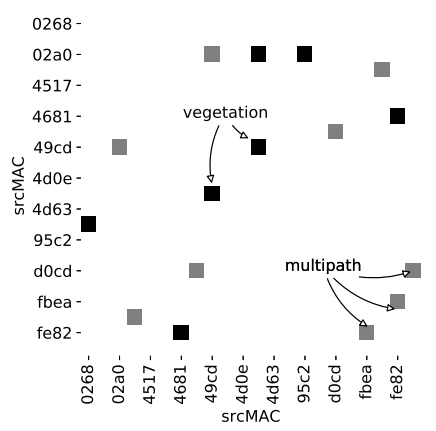

(d) Topography data and antenna pattern: $9(1.9 \%)$ in testbed but not in simulation, $7(1.4 \%)$ in simulation but not in testbed.

Fig. 5: Binary difference for simulations vs. testbed.

insights. Interference links measured in the testbed but not present in the simulation are likely the results of multi-path propagation. This error mainly occurs in the urban areas of our testbed. Interference predicted in the simulation but not measurable in our testbed occurs most likely due to vegetation which is not part of our topography data.

\section{B. Interference analysis}

We provide further analysis of internal interference in the following using the verified simulation approach and the harvested measurement data.

Fig. 6 visualizes a boxplot of the interference power at each radio. The crosstalk within the enclosure represents the highest interference power for the majority of radios. Co-channel interference becomes significant at this signal level [20]. Therefore, a requirement for $\mathrm{CA}$ algorithms can be the assignment of a guard period between channels assigned to radios within the same enclosure. There are two possibilities to lower this interference from a practical point-of-view. First, further shielding between the Wi-Fi cards on the SBC. Second, integrate the Wi-Fi transmitter directly into the antenna and interconnect radios at a single location via cable-based networks.

The distribution of interference power at each radio varies. The same observation holds for the number of interfering links. While some radios only interfere with three other radios 


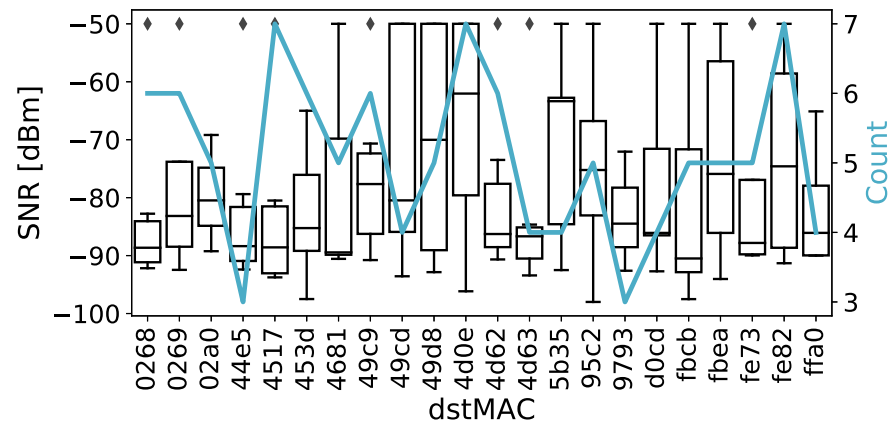

Fig. 6: Interference count and power distribution.

this values can increase up to seven. Therefore, to provide a network wide interference free channel allocation in our testbed at least seven different channels are needed. When additional factors such as external and co-channel interference are considered this number may be higher.

We noticed a low median of the interference power on some radios. The cumulative distribution function (CDF) in Fig. 7 visualizes this observation more clearly. More than $50 \%$ of the interference power measured fall below $-83 \mathrm{dBm}$ and more than $25 \%$ below $-91 \mathrm{dBm}$. One possibility is to set the CCA threshold of the Wi-Fi radios above e.g. $-83 \mathrm{dBm}$. Therefore, every signal below this level is treated as background noise not triggering a busy state at the Wi-Fi MAC layer. However, this exploit is only viable if the signal strength on the desired links is significantly higher than the new CCA threshold.

Distributed CA algorithms have advantages for dynamic topologies and changing interference conditions since certain events can be handled locally which reduces the decision delay [21]. However, only local information is taken into account usually represented by the n-hop neighborhood. We visualized the ratio of interfering links depended on the hop distance in Fig. 8 to assess the suitability of a distributed algorithm for our network architecture. The local crosstalkinterference (hop distance $=0$ ) accounts for $15 \%$. The highest amount of interference occurs at a hop distance of one. When two neighboring nodes have a common link using one of their multiple radios each, our results reveal a high probability for interference between the radio used for the link and the other radio on the neighboring node. For example, if two

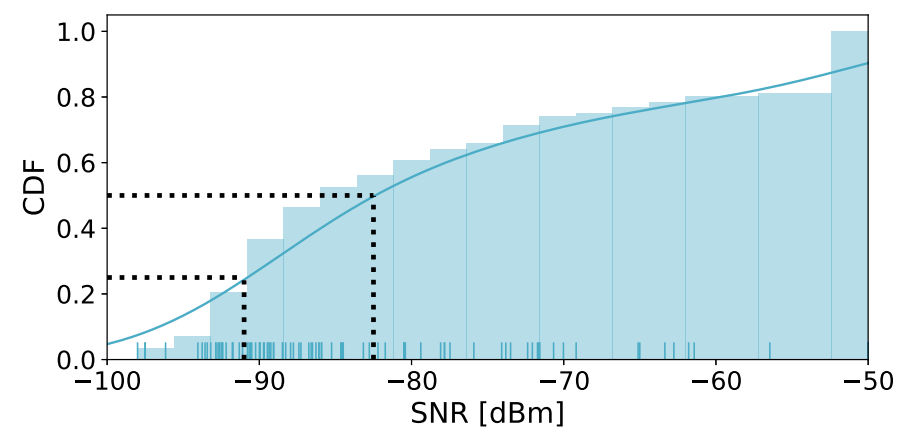

Fig. 7: Interference power empirical CDF.

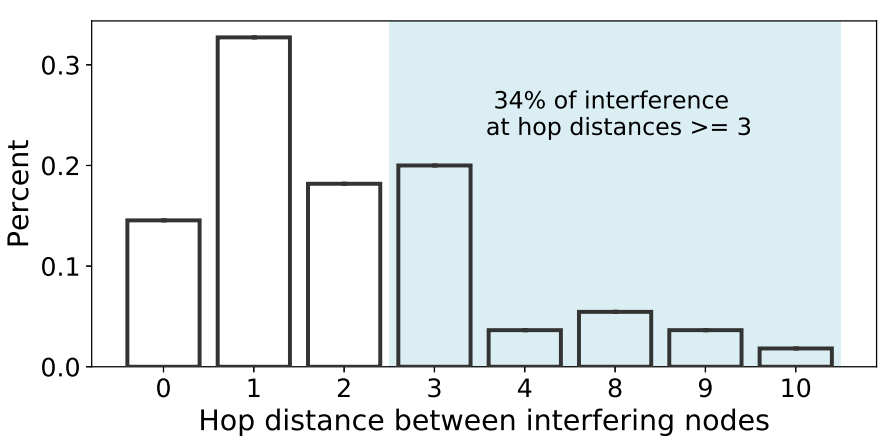

Fig. 8: Interference count and hop distances.

nodes $\mathrm{A}$ and $\mathrm{B}$ communicate via their radios $A 1 \leftrightarrow B 1$, there exists a significant chance for an interference between $A 1 \leftrightarrow B 2$ and $A 2 \leftrightarrow B 1$. One possibility to avoid this one hop distance interference is to install a local shielding between the antennas. While these two cases are included for distributed CA algorithms optimizing the 2-hop neighborhood, in our experiment $34 \%$ of interference occurs between nodes with a hop distance $>=3$. For real network deployments such as ours, distributed CA algorithms may not have sufficiently wide scope to find a near optimum channel allocation compared to centralized ones. The possibility that a distributed algorithm leads to a CA results far from global optimum is in particular undesirable for backhaul networks. In addition, due to the static nature of WiLD topologies, the main advantages of distributed algorithms (fast adaption to network changes and light-wide implementations) are not a mandatory criteria.

As described in Section III we conducted our measurements on different $20 \mathrm{MHz}$ channels in the $5 \mathrm{GHz}$ U-NII band successively using the same channel for all radios in the network. If a channel is switched by a CA algorithm, does the previous measured interference power change significantly? To estimate this aspect, Fig. 9 visualizes the overall interference power in the testbed for all different channels. The boxplot reveals that the interference median changes in the magnitude of a few decibels for different channels. In addition, no clear trend for the changes towards higher or lower frequencies is present. We are currently evaluating whether the differences are related to antenna gain or cable loss depending on the frequency.

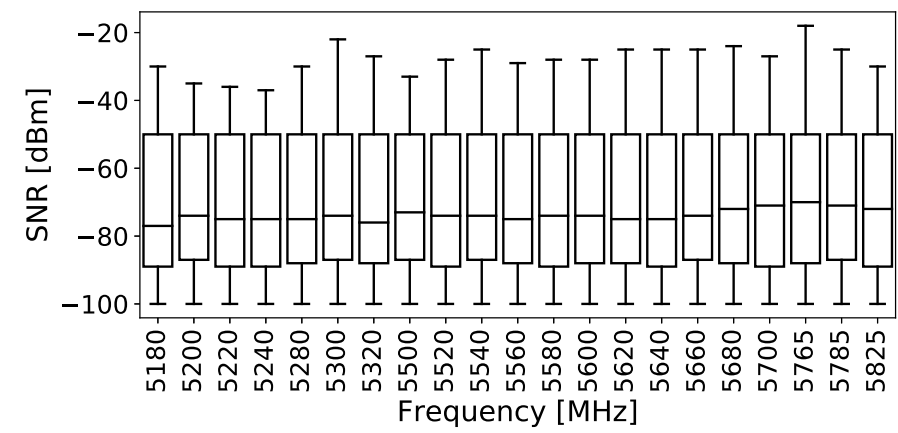

Fig. 9: Frequency dependency of interference. 


\section{CONCLUSiON}

In this work, we have described enhancements for a simulation model to quantify internal interference in WiLD networks. This work emphasizes that two extensions to previously used simulation models are needed. The main reduction of simulation errors was achieved by the usage of detailed antenna patterns compared to the widely used cone model. While the cone model is an easy way to mathematically model a directional antenna, it may significantly underestimate interference. A further improvement has been reached by using topography data instead of assuming line-of-sight propagation. Compared to previous work, the new enhanced simulation approach has been extensively verified by measurements from our testbed increasing the accuracy from $78 \%$ to $97 \%$. The source-code of the simulation is available in public domain [15].

Using the harvested data, we have provided insights to the origin of internal interference which can be used as foundations in the design phase of CA algorithms. A significant amount of interference in our testbed occurs between nodes with a hop-distance $>=3$. Therefore, considering network wide interference measurements as a source of information for a CA algorithm seems crucial. In addition, our measurement data reveals no significant difference in interference among different channels in the Unlicensed National Information Infrastructure (U-NII) band. The current multi-radio design of our WiLD nodes is beneficial for energy consumption and installation efforts. However, crosstalk between radios in the same enclosure is one of the main source of interference. Physical separation of the radios is one possibility to reduce this crosstalk-interference. Although, even with physically separated radios the crosstalk may occur between the local antennas effectively limiting the expected interference reduction.

\section{FUTURE WORK}

While our results were obtained with $5 \mathrm{GHz}$, exploring if a similar deviation between simulations and real-world measurements occurs in other frequency bands where directional antennas are used or proposed (e.g. mmWave) is an interesting future work item. In addition, we are also looking into the modeling of abstract antenna patterns such as the cone-plussphere model used in [22]. To further increase the accuracy and include obstacles such as small buildings and vegetation, LiDAR data [23] as a source for topography data may be worth exploring. The resolution is much higher compared to the SRTM data. However, LiDAR data is rarely available in public domain and the increased accuracy may lead to a significant increase in required processing power. Optimizing the CCA threshold to eliminate a certain amount of internal interference is a possibility worth exploring. However, it must be ensured that this optimization is compliant with current regulatory rules (listen before talk). In addition, there may be practical implications. When the Wi-Fi transmitter uses a higher CCA threshold, the demodulation of a signal on desired links could be impacted.

\section{REFERENCES}

[1] B. Sanou, "ICT Facts and 2016 figures," ICT Data and Statistics Division, Geneva, Tech. Rep., 2016.

[2] J. Saldana et al., "Alternative Networks: Toward a Global Access to the Internet for All," IEEE Commun. Mag., pp. 2-8, 2017.

[3] C. Niephaus et al., "Wireless back-haul: a software defined network enabled wireless back-haul network architecture for future $5 \mathrm{~g}$ networks," IET Networks, vol. 4, no. 6, pp. 287-295, 2015.

[4] F. Shzu-Juraschek, "Distributed channel assignment for multi-radio wireless mesh networks," PhD Thesis, Humboldt University of Berlin, 2014.

[5] M. Rademacher, K. Jonas, and M. Kretschmer, "Quantifying the spectrum occupancy in an outdoor $5 \mathrm{GHz}$ WiFi network with directional antennas," in 2018 IEEE Wirel. Commun. Netw. Conf. IEEE, apr 2018 pp. $1-6$.

[6] H.-N. Dai et al., "An overview of using directional antennas in wireless networks," Int. J. of Commun. Systems, vol. 26, no. 4, pp. 413-448, Apr 2013.

[7] E. Z. Tragos et al., "The impact of interference on the performance of a multi-path metropolitan wireless mesh network," Proc. IEEE Symposium on Computers and Commun., pp. 199-204, 2011.

[8] S. M. Das et al., "DMesh: Incorporating practical directional antennas in multichannel wireless mesh networks," IEEE J. Sel. Areas Commun., vol. 24, no. 11, pp. 2028-2039, 2006.

[9] W. Zhou, X. Chen, and D. Qiao, "Practical Routing and Channel Assignment Scheme for Mesh Networks with Directional Antennas," in IEEE Int. Conf. on Commun. IEEE, 2008, pp. 3181-3187.

[10] Q. Liu, X. Jia, and Y. Zhou, "Topology control for multi-channel multi-radio wireless mesh networks using directional antennas," Wireless Networks, vol. 17, no. 1, pp. 41-51, Jan 2011.

[11] N. Sadeghianpour, T. C. Chuah, and S. W. Tan, "Joint channel assignment and routing in multiradio multichannel wireless mesh networks with directional antennas," Int. Journal of Commun. Systems, vol. 28, no. 9, pp. 1521-1536, Jun 2015

[12] M. Xiao et al., "Millimeter Wave Communications for Future Mobile Networks," IEEE J. Sel. Areas Commun., vol. 35, no. 9, pp. 1909-1935, sep 2017.

[13] M. M. Carvalho and J. J. Garcia-Luna-Aceves, "Modeling Wireless Ad Hoc Networks with Directional Antennas," in Proc. IEEE INFOCOM. IEEE, 2006, pp. 1-12.

[14] M. Rademacher and K. Jonas, "Interference of simulated IEEE 802.11 links with directional antennas," in 2017 Wirel. Days, WD 2017. Porto: IEEE, mar 2017, pp. 27-32.

[15] M. Rademacher. (2019) Source-code. [Online]. Available: http://mclab.inf.h-brs.de/paper/wild-internal-interference/

[16] A. G. Longley and P. L. Rice, "Prediction of tropospheric radio transmission loss over irregular terrain. A computer method-1968," DTIC Document, Tech. Rep., 1968.

[17] M. Rademacher et al., "Experimental Results For the Propagation of Outdoor IEEE802.11 Links," VDE ITG-Fachbericht Mobilkommunikation, 2016.

[18] Earth Resources Observation and Science Center. (2019) SRTM [Online]. Available: https://dds.cr.usgs.gov/srtm/version1/

[19] B. Rabus et al., "The shuttle radar topography mission-a new class of digital elevation models acquired by spaceborne radar," ISPRS J. Photogramm. Remote Sens., vol. 57, no. 4, pp. 241-262, feb 2003.

[20] P. Fuxjager, D. Valerio, and F. Ricciato, "The myth of non-overlapping channels: interference measurements in IEEE 802.11," in 2007 Fourth Annu. Conf. Wirel. Demand Netw. Syst. Serv. IEEE, jan 2007, pp. 1-8.

[21] W. Si, S. Selvakennedy, and A. Y. Zomaya, "An overview of Channel Assignment methods for multi-radio multi-channel wireless mesh networks," J. of Parallel and Distributed Computing, vol. 70, no. 5, pp. 505-524, May 2010.

[22] V. Petrov et al., "Interference and SINR in Millimeter Wave and Terahertz Communication Systems With Blocking and Directional Antennas," IEEE Trans. Wirel. Commun., vol. 16, no. 3, pp. 1791-1808, mar 2017.

[23] S. Krishnan et al., "OpenTopography: A Services Oriented Architecture for Community Access to LIDAR Topography," in Proc. 2nd Int. Conf. Comput. Geospatial Res. Appl. - COM.Geo '11. New York, New York, USA: ACM Press, 2011, pp. 1-8. 